\title{
The true cost of host manipulation by parasites
}

Poulin, Robert; Fredensborg, Brian Lund; Hansen, Ellen Kathrine; Leung, Tommy L F

Published in:

Behavioural Processes

DOI:

10.1016/j.beproc.2004.07.011

Publication date:

2005

Citation for published version (APA):

Poulin, R., Fredensborg, B. L., Hansen, E. K., \& Leung, T. L. F. (2005). The true cost of host manipulation by parasites. Behavioural Processes, 68(3), 241-4. https://doi.org/10.1016/j.beproc.2004.07.011 


\title{
The true cost of host manipulation by parasites
}

\author{
Robert Poulin*, Brian L. Fredensborg, Ellen Hansen, Tommy L.F. Leung \\ Department of Zoology, University of Otago, P.O. Box 56, Dunedin, New Zealand \\ Received 27 July 2004; accepted 28 July 2004
}

Keywords: Fitness; Host defenses; Kin selection; Mortality risk; Non-host predators

If there is one thing that the past three decades of research in behavioural and evolutionary ecology have taught us, it is that there are no free lunches. Adaptive traits provide net fitness benefits to the animals bearing them, but the gains would be even greater if there were no concurrent costs associated with the expression of those traits. The ability of many parasites to enhance their transmission success by manipulating the behaviour of their hosts is one such trait (see Moore, 2002). In their excellent synthesis of past and current research on this phenomenon, Thomas et al. (2005) cast a doubt on the importance of the cost incurred by manipulating parasites. They argue that the existence and magnitude of such costs are dependent upon the type of mechanism used by a parasite to alter host behaviour. Thomas et al. (2005) focus exclusively on physiological, or proximate, costs such as the energetic costs necessary for the production of neuroactive substances in parasite species that use them to modify host behaviour. In a broader evolutionary context, however, one must consider costs at the ultimate level, in terms of fitness. Fitness is usually defined as the average number of sur-

\footnotetext{
* Corresponding author. Fax: +64 34797584.

E-mail address: robert.poulin@ stonebow.otago.ac.nz (R. Poulin).
}

viving offspring produced by individuals with a certain genotype relative to that produced by other genotypes, or as an individual's relative contribution of genes to future generations (Ridley, 1996; Freeman and Herron, 2001), Energy is not the best currency to measure loss of fitness, because other fitness components, such as mortality risk, cannot readily be quantified in units of energy. Elsewhere in their review, in their discussion of mafia-like strategies, Thomas et al. (2005) consider fitness costs, but a similar perspective is needed to assess the cost of manipulation itself.

Here, we wish to re-visit the issue of costs associated with manipulation. Even if the energetic expenses necessary to alter host behaviour are nil, a manipulative parasite may still incur a cost resulting, for instance, from a higher probability of being killed. This is a probabilistic cost, only paid in the event that the parasite dies, but it is a cost nonetheless. The net fitness outcome of manipulation must be positive, of course, otherwise it would not have been selected; it is the quantity subtracted from the gross benefits that we are dealing with here. Ideally, one would need to compare the fitness costs paid by a manipulative parasite with the costs, if any, incurred by a conspecific parasite that benefits from manipulation without itself inducing it. We found three examples of manipulative trematode 
species where such a contrast is possible. Thomas et al. (2005) question whether there is a cost associated with encystment in a particular site within the host as opposed to another site. Specifically, they ask whether it is costly for a parasite to encyst in the eye of a fish, referring to the well-studied trematodes such as Diplostomum spathaceum that encyst in the eye lenses of fish, altering the latter's behaviour and making them more susceptible to predation by avian definitive hosts (e.g., Crowden and Broom, 1980; Seppälä et al., 2004). The fact that a significant proportion of $D$. spathaceum cercariae die en route to the eyes after penetrating the fish through the gills or the skin (Ratanarat-Brockelman, 1974; Whyte et al., 1991) suggests that encysting in the eyes is costly. This is true even if the eye is a prime location for avoiding host immunity (the eye lens lacks blood vessels and circulating antibodies) and manipulating the host. Because all D. spathaceum cercariae migrate to the eyes of their fish host, it is not possible to compare them to conspecifics that encyst elsewhere. The three examples we chose allow such a comparison, and demonstrate that the site of encystment can relate to both the success of manipulation and its cost.

Our first example is the trematode Dicrocoelium dendriticum (and related species), the classical textbook example of a manipulative parasite. Infected ants, which serve as intermediate hosts for this trematode, attach to the tips of grass blades where they are presumably more susceptible to ingestion by grazing sheep, the parasite's definitive host (see Carney, 1969; Romig et al., 1980). Following their arrival in an ant, only one cercaria (the "brainworm") migrates to the ant's suboesophageal ganglion and induces the behavioural change; the others remain within the host's haemocoel. When an infected ant is eaten by a sheep, the brainworm invariably dies whereas the other parasites establish within the definitive host (Wickler, 1976). It appears that while it is inducing a change in host behaviour, the brainworm does not develop as do other metacercariae, and does not become infective for its next host. Thus, the manipulative individual dies whereas the non-manipulative individuals have a good chance of surviving and reproducing. Does that represent a cost of manipulation? We say it does.

It is possible that this cost in D. dendriticum is mitigated by kin selection. Based on the mode of transmission of this parasite, all cercariae ingested by a given ant may come from the same snail first intermediate host, i.e., they may all be derived from a single parasite egg. Cercariae shed from a snail occur as batches in slime balls exuded from the snail's mantle cavity. If a given ant feeds on a single slime ball containing several genetically identical cercariae, then the brainworm's sacrifice would ensure the transmission of its clones, and could be favoured by selection (Wickler, 1976; Wilson, 1977). In this scenario, manipulation would remain directly costly for the manipulative individual, but would be indirectly compensated by the increased success of the manipulator's kin. There is no confirmation, however, that $D$. dendriticum individuals inside the same ant are indeed clones; this is purely speculative at this point. In fact, there have been no studies on the genetic structure of groups of metacercariae sharing the same hosts in any of the many trematode species known to induce changes in the behaviour of intermediate hosts. In any event, the following two examples are set in aquatic habitats, where the dispersal of cercariae makes it extremely unlikely that clones regularly co-occur in the same intermediate host.

Our second example is the trematode Microphallus papillorobustus, another well-known manipulator. Metacercariae of this species that encyst in the cerebral region of the second intermediate host, the amphipod Gammarus aequicauda, induce a strong positive phototaxis and aberrant evasive responses in the host (Helluy, 1983). This manipulation of host behaviour results in infected amphipods being more susceptible to predation by aquatic birds, which serve as definitive hosts for the parasite (Helluy, 1984). However, not all metacercariae of M. papillorobustus encyst in the cerebral region of amphipods, some also encyst in the abdomen. Amphipods are capable of mounting an immune response against invading parasites, involving both encapsulation and melanization, and they use this cellular defense reaction against metacercariae. Thomas et al. (2000) have found that approximately $17 \%$ of cerebral metacercariae of $M$. papillorobustus are killed by encapsulation and melanization, whereas less than $1 \%$ of abdominal metacercariae suffer this fate. Three other trematode species, belonging to the same family (Microphallidae) as M. papillorobustus, also parasitize the amphipod G. aequicauda; they all encyst in the amphipod's abdomen, and none of them is attacked by the host immune system (Thomas et al., 2000). The host's defenses are apparently targeted specifically at those metacercariae most likely to cause it harm. The end 
result is that in this system, manipulative parasites incur a much greater probability of death from immune attack than their conspecifics opting not to manipulate and encysting in the abdomen of the host. Is manipulation costly in this trematode species? Here too, we say it is.

Our final example involves the two echinostome trematodes Curtuteria australis and Acanthoparyphium sp., which live in sympatry on New Zealand intertidal mudflats and have the same life cycle. After their release from snail first intermediate hosts, cercariae of both species penetrate a bivalve second intermediate host, the cockle Austrovenus stutchburyi, through its inhalant siphon; they encyst as metacercariae in the foot of cockles and await predation by oystercatchers, their common definitive host. As metacercariae accumulate in the foot of a cockle, they replace host muscle tissue and stunt further foot growth. The outcome is that heavily-parasitized cockles lose their ability to burrow, and are left stranded on the sediment surface (Thomas and Poulin, 1998). Two independent field experiments have shown that manipulated cockles are between 5 and 7 times more likely to be eaten by bird definitive hosts than healthy, buried cockles (Thomas and Poulin, 1998; Mouritsen, 2002). Both trematode species appear to contribute equally to this manipulation: their relative abundances vary from one locality to the next, but on a regional scale they are roughly equally abundant (Babirat et al., 2004). In addition, they both tend to encyst near the tip of the foot (Babirat et al., 2004), where their debilitating effect on the host's burrowing ability is most intense (Mouritsen, 2002). Importantly, however, about $25 \%$ of metacercariae of both species are found in the middle of the foot, and about 10\% near the base of the foot (Babirat et al., 2004). The benefits of host manipulation are shared by all metacercariae: although only those near the tip of the foot impair the burrowing ability of cockles, once an oystercatcher feeds on a cockle, it ingests all parasites along with host tissues. Predatory whelks also pick on surface-stranded cockles (Ansell, 2001; McFarland et al., 2003); although they only attack a small proportion of cockles, they ingest all tissues, and thus all parasites face the same risk whatever their exact site of encystment. Another opportunistic predator also picks on surface-stranded cockles, but this one eats exclusively the tip of the foot of surface-stranded cockles trying in vain to burrow. This predator, a labrid fish, is not a suitable definitive host for the two echinostome trematodes: any metacercaria ending up in a fish dies. Up to one-third of all cockles show signs that part of their foot has been cropped by the fish (Mouritsen and Poulin, 2003a). The frequency of foot cropping is particularly high among manipulated cockles lying on the sediment surface, and field estimates indicate that close to one-fifth of metacercariae are thus lost to fish predation (Mouritsen and Poulin, 2003b). All of those were encysted near the tip of the foot of cockles, not at its base. Thus, in this system, all metacercariae in a cockle have the same probability of reaching a bird definitive host, but those that are responsible for manipulating host behaviour face a greater risk of mortality. Is host manipulation costly in these two trematode species? Once again, we say it is.

The reader may feel that our three examples represent exceptions rather than the rule. To some extent, this is true: the host-parasite systems discussed above are unusual. But their uniqueness does not come from the fact that manipulation is costly in all of them, but instead from the fact that they allow this cost to be estimated. Intraspecific variation in whether or not parasite individuals contribute to host manipulation (i.e., intraspecific variation in site of encystment within the host) is the common feature of these systems, one that provides a baseline for measuring fitness costs associated with manipulation. Other manipulative parasite species do not display any such variation, or if they do it has not been examined or reported. It is thus the existence of cheaters, who benefit from manipulation without incurring its cost, that allow us to estimate this cost in our three examples. In addition, the three families (Dicrocoeliidae, Microphallidae and Echinostomatidae) involved in our examples fall on completely different branches of the phylogeny of trematodes (Olson et al., 2003); the cost of host manipulation is therefore not restricted to a single trematode clade.

Thomas et al. (2005) presented a proximate-level view of the cost of host manipulation incurred by manipulative parasites. Our main purpose in this commentary was to complement their review and offer an alternative, and somewhat broader, perspective of the costs of manipulation. The proximate physiological costs of manipulation, measured in terms of the energy expended by the parasite, may often be trivial, but the ultimate fitness costs paid by manipulative parasites may still be considerable. Similarly, the costs of defenses 
against parasites incurred by resistant hosts may also appear small when seen from a metabolic perspective, but their fitness costs are often substantial (Sheldon and Verhulst, 1996; Lochmiller and Deerenberg, 2000). The examples we have chosen illustrate that manipulation of the host can result in higher mortality risks for manipulative individuals compared to their nonmanipulative conspecifics. The actual sources of parasite mortality vary among host-parasite systems, from host immune responses to predation by non-host predators. Fitness costs involving reduced future fecundity instead of higher risk of mortality are also possible. Of course, the net outcome of manipulation, i.e., the difference between fitness gains and fitness costs, taking into account that both gains and costs are only probabilistic (higher probability of transmission, higher risk of death from various causes), must be positive for manipulation to evolve. This does not mean that the cost is negligible, however. In fact, high costs may be one key reason why host manipulation is observed in some parasite species, but not in other closely-related species.

\section{References}

Ansell, A.D., 2001. Dynamics of aggregations of a gastropod predator/scavenger on a New Zealand harbour beach. J. Mollusc. Stud. 67, 329-341.

Babirat, C., Mouritsen, K.N., Poulin, R., 2004. Equal partnership: two trematode species, not one, manipulate the burrowing behaviour of the New Zealand cockle, Austrovenus stutchburyi. J. Helminthol. 78, 195-199.

Carney, W.P., 1969. Behavioral and morphological changes in carpenter ants harboring dicrocoeliid metacercariae. Am. Midl. Nat. 82, 605-611.

Crowden, A.E., Broom, D.M., 1980. Effects of the eyefluke, Diplostomum spathaceum, on the behaviour of dace (Leuciscus leuciscus). Anim. Behav. 28, 287-294.

Freeman, S., Herron, J.C., 2001. Evolutionary Analysis, second ed. Prentice Hall, New Jersey.

Helluy, S., 1983. Relations hôtes-parasites du trématode Microphallus papillorobustus (Rankin 1940). II. Modifications du comportement des Gammarus hôtes intermédiaires et localisation des métacercaires. Ann. Parasitol. Hum. Comp. 58, 1-17.

Helluy, S., 1984. Relations hôtes-parasites du trématode Microphallus papillorobustus (Rankin 1940). III. Facteurs impliqués dans les modifications du comportement des Gammarus hôtes in- termédiaires et tests de prédation. Ann. Parasitol. Hum. Comp. $59,41-56$.

Lochmiller, R.L., Deerenberg, C., 2000. Trade-offs in evolutionary immunology: just what is the cost of immunity? Oikos 88, 87-98.

McFarland, L.H., Mouritsen, K.N., Poulin, R., 2003. From first to second and back to first intermediate host: the unusual transmission route of Curtuteria australis (Digenea: Echinostomatidae). J. Parasitol. 89, 625-628.

Moore, J., 2002. Parasites and the Behavior of Animals. Oxford University Press, Oxford.

Mouritsen, K.N., 2002. The parasite-induced surfacing behaviour in the cockle Austrovenus stutchburyi: a test of an alternative hypothesis and identification of potential mechanisms. Parasitology 124, 521-528.

Mouritsen, K.N., Poulin, R., 2003a. The risk of being at the top: footcropping in the New Zealand cockle Austrovenus stutchburyi. J. Mar. Biol. Assoc. UK 83, 497-498.

Mouritsen, K.N., Poulin, R., 2003b. Parasite-induced trophic facilitation exploited by a non-host predator: a manipulator's nightmare. Int. J. Parasitol. 33, 1043-1050.

Olson, P.D., Cribb, T.H., Tkach, V.V., Bray, R.A., Littlewood, D.T.J., 2003. Phylogeny and classification of the Digenea (Platyhelminthes: Trematoda). Int. J. Parasitol. 33, 733-755.

Ratanarat-Brockelman, C., 1974. Migration of Diplostomum spathaceum (Trematoda) in the fish intermediate host. Z. Parasitenk. 43, 123-134.

Ridley, M., 1996. Evolution, second edition. Blackwell Science, Oxford.

Romig, T., Lucius, R., Frank, W., 1980. Cerebral larvae in the second intermediate host of Dicrocoelium dendriticum (Rudolphi, 1819) and Dicrocoelium hospes Looss, 1907 (Trematoda, Dicrocoeliidae). Z. Parasitenk. 63, 277-286.

Seppälä, O, Karvonen, A., Valtonen, E.T., 2004. Parasite-induced change in host behaviour and susceptibility to predation in an eye fluke-fish interaction. Anim. Behav. 68, 257-263.

Sheldon, B.C., Verhulst, S., 1996. Ecological immunology: costly parasite defences and trade-offs in evolutionary ecology. Trends Evol. Ecol. 11, 317-321.

Thomas, F., Adamo, S., Moore, J., 2005. Parasitic manipulation: where are we and where should we go? Behav. Process. 68, 185199.

Thomas, F., Guldner, E., Renaud, F., 2000. Differential parasite (Trematoda) encapsulation in Gammarus aequicauda (Amphipoda). J. Parasitol. 86, 650-654.

Thomas, F., Poulin, R., 1998. Manipulation of a mollusc by a trophically transmitted parasite: convergent evolution or phylogenetic inheritance? Parasitology 116, 431-436.

Whyte, S.K., Secombes, C.J., Chappell, L.H., 1991. Studies on the infectivity of Diplostomum spathaceum in rainbow trout (Oncorhynchus mykiss). J. Helminthol. 65, 169-178.

Wickler, W., 1976. Evolution-oriented ethology, kin selection, and altruistic parasites. Z. Tierpsychol. 42, 206-214.

Wilson, D.S., 1977. How nepotistic is the brain worm ? Behav. Ecol. Sociobiol. 2, 421-425. 